University of Nebraska - Lincoln

DigitalCommons@University of Nebraska - Lincoln

Faculty Publications in Computer \& Electronics Electrical \& Computer Engineering, Department Engineering (to 2015)

2011

\title{
Distributed MIMO Technologies in Cooperative Wireless Networks
}

\author{
Shichuan $\mathrm{Ma}$ \\ University of Nebraska-Lincoln \\ Yaoqing (Lamar) Yang \\ University of Nebraska-Lincoln \\ Hamid Sharif \\ University of Nebraska-Lincoln, hsharif@unl.edu
}

Follow this and additional works at: https://digitalcommons.unl.edu/computerelectronicfacpub

Part of the Computer Engineering Commons

Ma, Shichuan; (Lamar) Yang, Yaoqing; and Sharif, Hamid, "Distributed MIMO Technologies in Cooperative Wireless Networks" (2011). Faculty Publications in Computer \& Electronics Engineering (to 2015). 80. https://digitalcommons.unl.edu/computerelectronicfacpub/80

This Article is brought to you for free and open access by the Electrical \& Computer Engineering, Department of at DigitalCommons@University of Nebraska - Lincoln. It has been accepted for inclusion in Faculty Publications in Computer \& Electronics Engineering (to 2015) by an authorized administrator of DigitalCommons@University of Nebraska - Lincoln. 


\title{
Distributed MIMO Technologies in Cooperative Wireless Networks
}

\author{
Shichuan Ma, Yaoqing (Lamar) Yang, and Hamid Sharif, University of Nebraska-Lincoln
}

\begin{abstract}
Multiple-input multiple-output techniques enhance wireless communications performance by taking advantage of spatial diversity. However, most traditional MIMO systems hardly achieve large spatial diversity because of limited terminal size. In this article, we present recent advances of the distributed MIMO technologies in cooperative wireless networks. We also compare and discuss various relay protocols and cooperative strategies. Our simulation results indicate that distributed MIMO systems can provide larger spatial diversity, and the data rate in cooperative networks can be significantly increased.
\end{abstract}

\section{INTRODUCTION}

With ever increasing demands for multimedia services and web-related content, a high data rate is becoming one of the major features in the next generation of wireless communication systems. However, channel fading, an inherent property of wireless communication links, severely limits the increase of the data rate [1]. The most common and effective way to combat channel fading is to exploit the diversity from the received signals. By transmitting a signal via multiple independent channels, such as at different time slots, in different frequency bands, and from different spatial directions, the receiver can receive different copies of the signal and thus achieve time, frequency, and space diversity gains by employing optimal combining schemes.

Spatial diversity techniques are particularly attractive since they provide diversity gain without incurring extra costs of transmission time and bandwidth. Traditionally, spatial diversity is achieved by using multiple antennas at the transmitter and/or receiver, where the antennas are packed together with spacing on the order of a wavelength, referred as collocated multiple-input multiple-output (MIMO). Because of the diversity gain, collocated MIMO architectures are effective in increasing system throughput and capable of combating channel fading.

However, the benefits of the collocated MIMO technique are limited in practical systems [2]. The reasons for this limitation are twofold. First, spatial correlation causes performance degradation. In a collocated MIMO system, antennas at each node have to be placed close to each other. Thus, radio signals at the collocated antennas experience a similar scattering environment, and the channels may be correlated, especially when a line-of-sight (LOS) channel between the transmitter and receiver dominates. The channel matrices could be ill conditioned, resulting in significant capacity decrease. Second, due to the terminal size limitation, the node cannot be equipped with many antennas. Since the diversity gain is proportional to the number of antennas, a collocated MIMO system with few antennas cannot produce the expected performance.

To mitigate the aforementioned drawbacks in collocated MIMO systems, a new technique named distributed MIMO was proposed and has attracted much attention [3]. The major difference between the distributed and collocated MIMO is that multiple antennas at the front end of wireless networks are distributed among widely separated radio nodes. In a distributed MIMO system, each node may be only equipped with one antenna. Many nodes at different locations transmit the same information to the receiver. In this manner, multiple nodes form a virtual antenna array that achieves higher spatial diversity gain. This kind of spatial diversity is referred to as user cooperation diversity [3], or simply cooperative diversity [4].

In this article, we provide a detailed study of the recent advances in distributed MIMO technologies in cooperative wireless networks. Particularly, we concentrate on physical layer techniques instead of the design of higher layers. This article is organized as follows. We introduce the basic concept of a cooperation system. Next, we discuss relay protocols and cooperative strategies. We present simulation results that show the effectiveness of a cooperative network with full user cooperation diversity and improved bandwidth efficiency. At the end, we conclude this article.

\section{COOPERATION SYSTEMS}

A cooperative wireless network consists of a source, a number of relays, and a destination, which is illustrated in Fig. 1. Because we deal with physical layer techniques instead of higher layer protocols in this article, it is reasonably assumed in the network that all nodes use the same multiple access resources; for example, they use the same subchannel in a frequency-division multiple access (FDMA) system. Therefore, the network 
in Fig. 1 is a subset of a practical network. It could be a cell in a cellular system or a same-frequency cluster in a mesh network.

In Fig. 1, the source node intends to transmit information to destination node $D$. Other nodes in the network could be selected as helpers or relays for the transmission between the source and the destination. Assuming that nodes $R_{1}, R_{2}$, $\cdots$, and $R_{n}$ are selected to be the helpers, we treat these helpers as relay nodes, which forward the signals from the source to the destination. The relays together with the source form a virtual transmit array to achieve spatial diversity. Relays in a cooperation network are different from those in a relay network where the relays only forward signals from other nodes. In cooperation networks, any nods can function as a source or a relay. When a node collects and transmits information, it works as a source. Otherwise, it can help other nodes as a relay.

When a communication link is established for transmitting information, relay nodes are selected based on a set of rules or relay selection strategies. Usually metrics such as the locations, loads, and end-to-end performance of the relays are considered in a network. To complete this task, a cross-layer design is employed, which is beyond the scope of this article. Interested readers can refer to an excellent article [5] for details.

The communication between the source and destination proceeds in two phases: information sharing and cooperative transmission. In the information sharing phase, the source broadcasts its information to the relays and the destination. This step is inevitable because spatial diversity can only be achieved from independent transmission of the same information. Through information sharing, all relays get the information from the source and enable independent data transmission. In the second phase, the information is forwarded by the relays to the destination. In the meantime, the source could either transmit or remain inactive. Because the relays are randomly selected and are usually separated far away from others, it becomes a strong likelihood that the channels between each relay and the destination are uncorrelated. In cooperative transmission, multiple relays and a source node (each of which is equipped with a single antenna) form a virtual antenna array for a distributed MIMO system. This distributed MIMO provides the benefits of the cooperative system by overcoming the size limitation and ill conditioned channel matrix in collocated MIMO systems.

Co-channel interference is a serious problem in a cooperative wireless network. When the information is relayed to the destination, there exists co-channel interference in that the signals from different relays may interfere with each other at the destination. Although the interference cancellation at the destination is a possible solution, the required algorithm is complex, and the performance may not be very satisfying. The common cooperative strategy is to avoid interference by transmitting the relayed signals in orthogonal subchannels. The orthogonality could be acquired by using a repetition-based strategy or distributed space-time coding (DSTC). For the repetition-based strategy, the relays forward the signal in different time slots; that is, in each

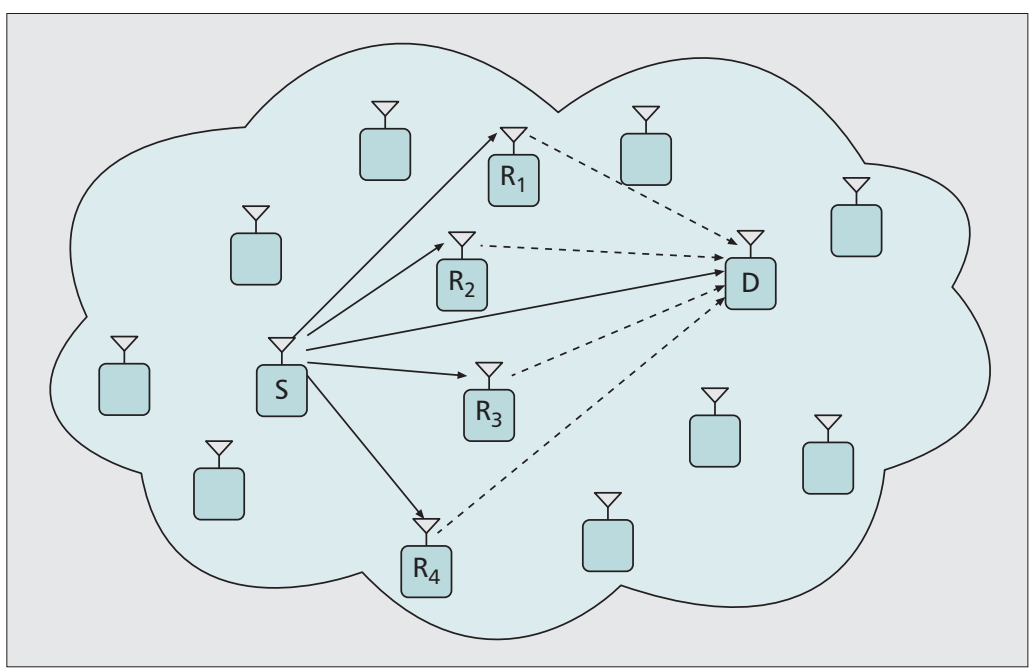

Figure 1. A cooperative wireless network.

time slot, only one relay transmits information, while the other relays stay inactive. This strategy is easy to implement, but results in poor bandwidth efficiency. By using DSTC, the relays can forward information in the same time slot. The orthogonality is constructed in both time and space domains. Although the DSTC-based strategy leads to a more complex network, the bandwidth is utilized more efficiently.

Both repetition-based and DSTC-based cooperative strategies can achieve full spatial diversity (i.e., the $n+1$ order of spatial diversity). However, the achieved diversity is not only decided by the cooperative strategies, but also determined by the relay protocols, which define the relaying methods for the relays. We first discuss relay protocols in the next section, and then compare the cooperative strategies later.

\section{RELAY PROTOCOLS}

Relay protocols significantly affect the system performance in cooperative wireless networks. In this section, we present an overview of commonly used relay protocols.

\section{AMPLIFY-AND-FORWARD}

The simplest relay protocol is amplify-and-forward (AF) [6]. In AF, each relay amplifies the received noisy signals and forwards them to the destination. This simple processing benefits cooperative wireless networks with full spatial diversity at high signal-to-noise ratios (SNRs). Because the noise component is also amplified in $\mathrm{AF}$, the bit error rate (BER) performance could be degraded. To optimize the performance in $\mathrm{AF}$ systems, a scalar used for amplification is chosen adaptively based on the channel coefficient.

\section{DECODE-AND-FORWARD}

For decode-and-forward (DF) relay protocol, the relays first decode the received signals and then forward the re-encoded signals to the destination node [7]. The decoding can be done fully in bit level or partially in symbol level. When the channel between the source and the relay is of good quality, DF provides error correlation capability and is then superior to AF. However, 


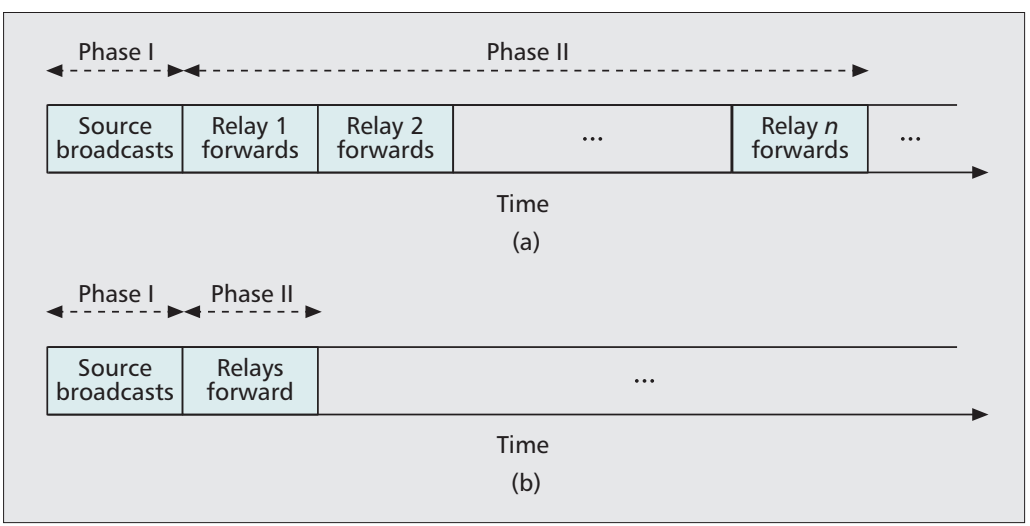

Figure 2. Slot assignment for cooperation strategies: a) repetition-based; $b$ ) DSTC-based. single bit to the source and relays to indicate the success or failure of the direct transmission. If the direct transmission succeeds, the relays will not forward the signals, and the source will continue to the next time slot of new information transmission. Otherwise, relays forward the signals to the destination. This protocol improves bandwidth utilization only at high SNRs.

\section{DSTC-BASEd CoOperative Strategy}

Repetition-based cooperative diversity algorithms can achieve full spatial diversity at the expense of decreasing bandwidth efficiency. The utilization of incremental relaying cannot overcome this drawback when the source-destination link is in poor condition. To further improve bandwidth efficiency, DSTC can be used to enable relays to transmit in the same time slot in cooperative systems [9]. In the DSTC-based cooperative strategy the source broadcasts information in phase one. Contrary to the above repetition-based cooperative strategy, the source and all relays simultaneously transmit coded signals in phase two. Thus, we can achieve the full cooperative diversity gain, $n+1$.

DSTC-based cooperative strategy is usually applied together with AF or DF relay protocol. After the signals are amplified or decoded, the relays will re-encode the signal before forwarding. Different from the DF protocol, the signals are re-encoded by using distributed space-time codes in the DSTC-based strategy. Distributed spacetime codes can be directly obtained from conventional space-time codes such that each code is applied to the antenna at each node. Commonly used distributed space-time codes are distributed space-time block codes and distributed space-time trellis codes. These codes guarantee that the signals from different relays are orthogonal, and hence can be separated at the destination without interference. In this manner, all relays forward the signals at the same time slot, as shown in Fig. $2 \mathrm{~b}$. Comparing Fig. $2 \mathrm{a}$ and $2 \mathrm{~b}$, it is found that the channel utilization of the DSTC-based strategy is better than that of the repetition-based strategy.

The bandwidth efficiency improvement provided by the DSTC-based strategy comes at the cost of complex signaling and signal processing. To implement DSTC, the relays should have a priori knowledge of the space-time codes assigned to them. This requires extra signaling over dedicated channels. However, the bandwidth for the extra signaling is negligible compared to the improved bandwidth utilization. While space-time coding adds some computation complexity to the relays, the one-antenna design simplifies the relays significantly. Consequently, the nodes in a cooperative wireless network are relatively simple compared to those in a traditional MIMO system, which are mounted with multiple antennas and have to process signals from multiple physical channels.

\section{Performance Comparisons}

In this section we present our simulation results and compare the performance of cooperative wireless networks with different relay protocols and cooperative strategies. A flat fading channel is used in the simulations. In [8], Laneman et al. analyzed bandwidth efficiency by studying the 
outage probability $\operatorname{Pr}[I<R]$; that is, given the channel realization, the probability of the mutual information $I$ is less than a given data rate $R$. The outage probabilities for different relay protocols are compared in Fig. 3, where the $\mathrm{x}$-axis is the SNR and the $y$-axis is the outage probability $P_{\text {outage }}$. The data rate is set to $R=1 \mathrm{~b} / \mathrm{s} / \mathrm{Hz}$, and the number of relay $n=1$ in this scenario. Compared to direct transmission, the $\mathrm{AF}$, selection DF, and incremental AF protocols can achieve full diversity gain ( 2 in this scenario) because their curves are nearly double as steep as that of direct transmission. The incremental AF protocol performs even better since the one-bit feedback can effectively reduce the relay repetitions. For example, the incremental AF protocol achieves about $17 \mathrm{~dB}$ gain over direct transmission at an outage probability of $10^{-3}$. The DF protocol, however, performs similarly to the direct transmission because it cannot achieve full spatial diversity. In this single relay scenario, the DF protocol cannot achieve any spatial diversity.

The outage capacities of the repetition-based cooperative strategy are shown and compared in Fig. 4, where the $\mathrm{x}$-axis is the data rate in bit per second per Hertz, and the y-axis is the outage probability $P_{\text {outage }}$. It is observed that the outage probabilities for the direct transmission and repetition-based cooperative strategy increase with the data rate. This is reasonable based on the definition of the outage probability. However, the relationship between the outage probability and the number of relays becomes complicated. At low data rates, the outage probability performance becomes better with more relays, while at high data rates, the outage probability becomes worse when more relays are used. For example, at 1 $\mathrm{b} / \mathrm{s} / \mathrm{Hz}$ data rate, the outage probability is $3 \times 10^{-4}$ for the one-relay scheme, and it improves to $2 \times$ $10^{-5}$ for the three-relay scheme. When the data rate is above $1.4 \mathrm{~b} / \mathrm{s} / \mathrm{Hz}$, however, the outage probability of the three-relay scheme is higher than that of the one-relay scheme. This phenomenon results from the poor bandwidth efficiency of the repetition-based cooperative strategy. From the above analysis, we realize that the repetition-based cooperative strategy can guarantee a specific low data rate, but it cannot increase the data rate by adding more relays in the cooperative wireless network.

In contrast to the repetition-based cooperative strategy, the DSTC-based cooperative strategy offers better bandwidth efficiency, as shown in Fig. 5, where the x-axis is the data rate in bits per second per Hertz, and the y-axis is the outage probability $P_{\text {outage. }}$. When three relays are used, the outage probability is about $10^{-7}$ at 1 $\mathrm{b} / \mathrm{s} / \mathrm{Hz}$, which is much lower than the outage probability $2 \times 10^{-5}$ of the repetition-based cooperative strategy. This bandwidth utilization improvement results from the simultaneous transmission of a group of relays. Due to the same cause, adding more relays will not decrease the data rate. On the contrary, more relays will increase the data rate due to the higher order of spatial diversity. For example, in Fig. 5, comparing the three-relay scheme to the one-relay scheme, the outage probability becomes lower by about an order of two. It should be noted that the achievable data rate cannot exceed the channel capacity. Therefore, the curves in Fig. 5 will

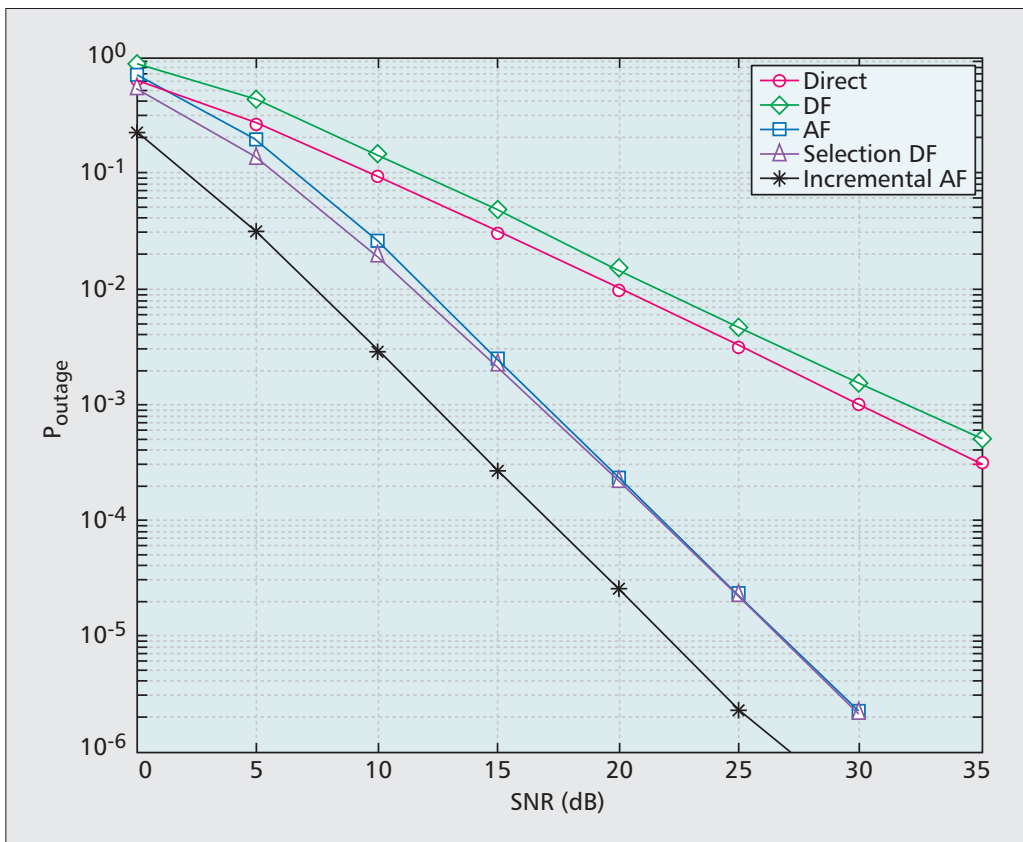

Figure 3. Performance comparisons of different relay protocols. One relay is used and data rate is set to $1 \mathrm{~b} / \mathrm{s} / \mathrm{Hz}$.

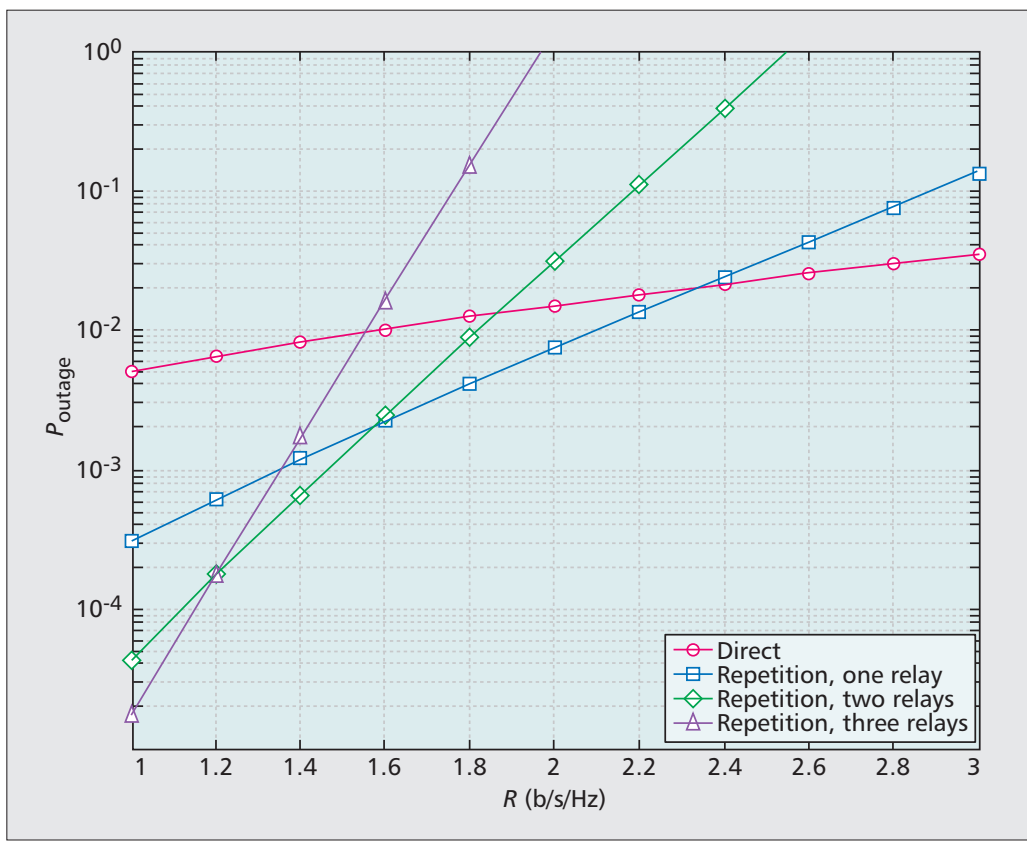

Figure 4. Outage probabilities for repetition-based cooperative diversity. Results of $\mathrm{n}=1,2$, and 3 relays are shown. The signal-to-noise ratio is set to $20 \mathrm{~dB}$.

converge at $P_{\text {outage }}$ for sure when the data rate is greater than the channel capacity.

Finally, the BER performance of repetitionbased and DSTC-based cooperative strategies is shown in Fig. 6, where the $\mathrm{x}$-axis is the SNR in $\mathrm{dB}$ and the $y$-axis is the average BER. The code used in the simulation is single-symbol maximum likelihood decodable distributed STBC proposed in [10]. Three relays and AF relay protocol are used in the simulation. Given the same data rate, the DSTC-based strategy outperforms repetitionbased strategy in terms of average BER. For example at $2 \mathrm{~b} / \mathrm{s} / \mathrm{Hz}$ in Fig. 6, the DSTC-based 
cooperative strategy acquires about $7 \mathrm{~dB}$ gain over the repetition-based cooperative strategy at $10^{-4}$ average BER. When the data rate increases, the BER performance of both strategies degrades. Especially, it is observed that the degradation for the repletion-based strategy is more severe. For instance, at average BER $10^{-4}$, the BER degradation from $2 \mathrm{~b} / \mathrm{s} / \mathrm{Hz}$ to $1 \mathrm{bt} / \mathrm{s} / \mathrm{Hz}$ is about $5 \mathrm{~dB}$ for the DSTC-based cooperative strategy but about $10 \mathrm{~dB}$ for the repetition-based cooperative strategy. This simulation result shows again that the repetition-based strategy is bandwidth inefficient.

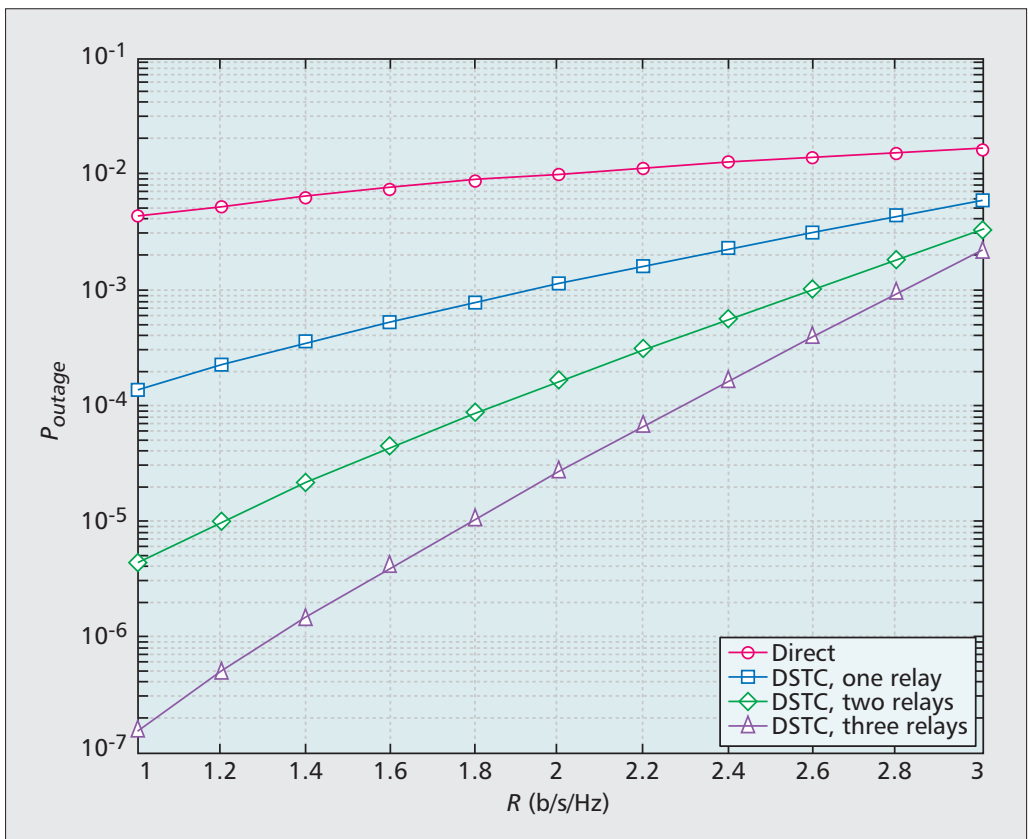

Figure 5. Outage probabilities for DSTC-based cooperative diversity. Results for $\mathrm{n}=1,2$, and 3 relays are shown. The SNR is set to $20 \mathrm{~dB}$.

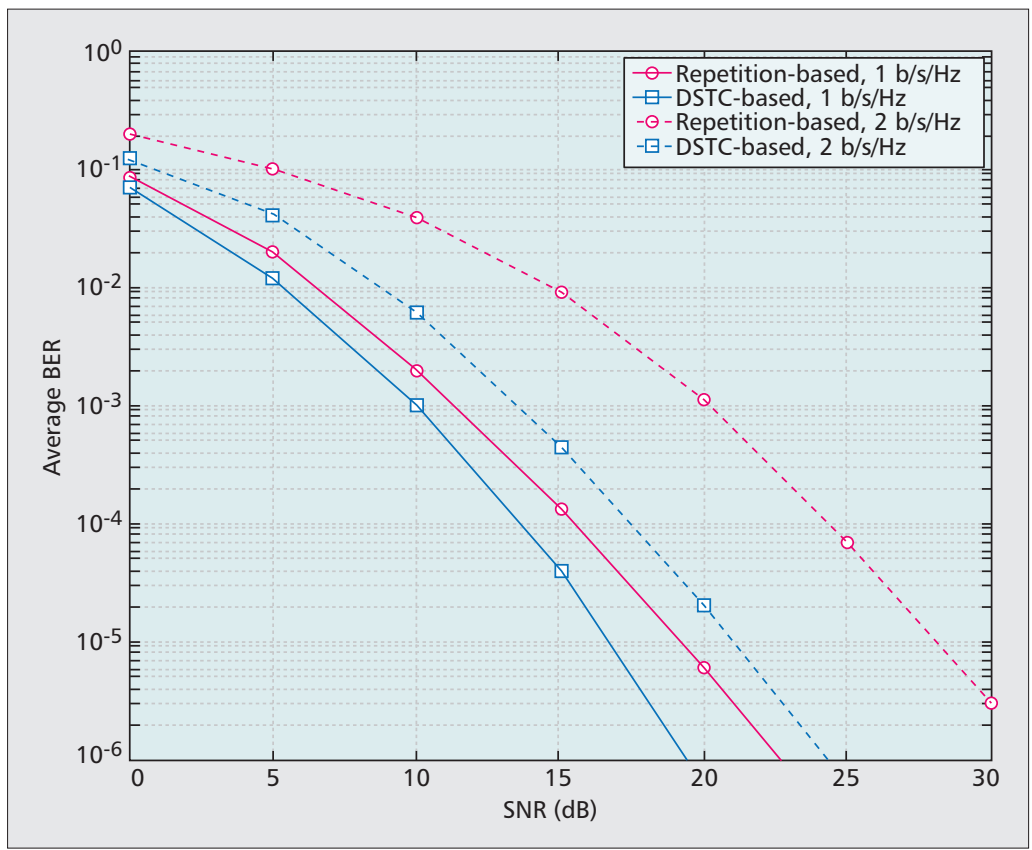

Figure 6. Bit error rate performance comparison of repetition-based and DSTCbased cooperative strategies. Amplify-and-forward relay protocol and $\mathrm{n}=2$ relays are used in simulation.

\section{CONCLUSIONS}

In this article, we present a detailed discussion of the latest distributed MIMO technologies in cooperative wireless networks. This includes the principle of cooperative wireless communications, and popular relay protocols and cooperative strategies. Our analysis incorporates a performance comparison of outage probability and bit error rates. The simulation results indicate that distributed MIMO can provide full user cooperation diversity, and the data rate in cooperative networks can be significantly increased by using distributed spacetime coding.

\section{REFERENCES}

[1] A. Paulraj, R. Nabar, and D. Gore, Introduction to Space-Time Wireless Communications, Cambridge Univ. Press, 2003.

[2] E. A. Jorswieck and H. Boche, "Optimal Transmission Strategies and Impact of Correlation in Multiantenna Systems with Different Types of Channel State Information," IEEE Trans. Sig. Proc., vol. 52, no. 12, Dec. 2004.

[3] A. Sendonaris, E. Erkip, and B. Aazhang, "User Cooperation Diversity - Part I \& II," IEEE Trans. Commun., vol. 51, no. 11, Nov. 2003, pp. 1927-48.

[4] V. Stankovic, A. Host-Madsen, and Z. Xiong, "Cooperative Diversity for Wireless Ad Hoc Networks," IEEE Sig. Proc., vol. 23, no. 5, Sept. 2006, pp. 37-49.

[5] P. Liu et al., "Cooperative Wireless Communications: A Cross-Layer Approach," IEEE Wireless Commun., vol. 13, no. 4, Aug. 2006, pp. 84-92.

[6] T. Issariyakul and V. Krishnamurthy, "Amplify-and-Forward Cooperative Diversity Wireless Networks: Model, Analysis, and Monotonicity Properties," IEEE/ACM Trans. Net., vol. 17, no.1, Feb. 2009, pp. 225-38.

[7] K.-S. Hwang, Y.-C. Ko, and M.-S. Alouini, "Outage Probability of Cooperative Diversity Systems with Opportunistic Relaying Based on Decode-and-Forward," IEEE Trans. Wireless Commun., vol. 7, no. 12, Dec. 2008, pp. 5100-07.

[8] J. N. Laneman, D. N. C. Tse, and G. W. Wornell, "Cooperative Diversity in Wireless Networks: Efficient Protocols and Outage Behavior," IEEE Trans. Info. Theory vol. 50, no. 12, Dec. 2004, pp. 3062-80.

[9] Y. Jing and B. Hassibi, "Distributed Space-Time Coding in Wireless Relay Networks," IEEE Trans. Wireless Commun., vol. 5, no. 12, Dec. 2006, pp. 3524-36.

[10] Z. Yi and I. M. Kim, "Single-Symbol ML Decodable Distributed STBCs for Cooperative Networks," IEEE Trans. Info. Theory, vol. 53, no. 8, Aug. 2007, pp. 2977-85.

\section{BIOGRAPHIES}

SHICHUAN MA (sma@huskers.unl.edu) received B.S. and M.S. degrees in information and communication engineering from Beijing University of Posts and Telecommunications, China, in 2000 and 2006, respectively. He is currently a Ph.D. candidate in computer and electronics engineering at the University of Nebraska-Lincoln. His research interests include MIMO, space-time coding, multicarrier modulation, and spread spectrum techniques for wireless communications.

YAOQING (LAMAR) YANG (yyang3@unl.edu) received a Ph.D. degree in the area of wireless communications and networks from the University of Texas at Austin in 2006. Since then, he has been an assistant professor in the Department of Computer and Electronics Engineering, University of Nebraska-Lincoln. His current research interests lie in wireless communications and networks, statistical signal processing, and cognitive radios.

HAMID SHARIF (hsharif@unl.edu) is the Paul and Betty Distinguished Professor in the Department of Computer and Electronics Engineering at the University of Nebraska-Lincoln (UNL). He is also director of the Advanced Telecommunications Engineering Laboratory (TEL) at UNL. He has authored/coauthored more than 170 articles in refereed national and international journals and conferences. He is currently co-Editor-in-Chief of John Wiley's Security and Communication Networks Journal. 